\title{
Higher Urinary Levels of 8-Hydroxy-2'-deoxyguanosine Are Associated with a Worse RANKL/OPG Ratio in Postmenopausal Women with Osteopenia
}

\author{
Carlo Cervellati, ${ }^{1}$ Arianna Romani, ${ }^{1}$ Eleonora Cremonini, ${ }^{2}$ \\ Carlo M. Bergamini, ${ }^{1}$ Enrica Fila, ${ }^{3,4}$ Monica Squerzanti, ${ }^{1}$ Pantaleo Greco, ${ }^{4}$ \\ Leo Massari, ${ }^{3,5}$ and Gloria Bonaccorsi ${ }^{3,4}$ \\ ${ }^{1}$ Department of Biomedical and Specialist Surgical Sciences, Section of Medical Biochemistry, \\ Molecular Biology and Genetics, University of Ferrara, Via Borsari 46, 44121 Ferrara, Italy \\ ${ }^{2}$ Department of Nutrition, University of California, One Shields Avenue, Davis, CA 95616, USA \\ ${ }^{3}$ Department of Morphology, Surgery and Experimental Medicine, Menopause and Osteoporosis Centre, University of Ferrara, \\ Via Boschetto 29, 44124 Ferrara, Italy \\ ${ }^{4}$ Department of Morphology, Surgery and Experimental Medicine, Section of Obstetrics and Gynecology, University of Ferrara, \\ Via Aldo Moro 8, Cona, 44124 Ferrara, Italy \\ ${ }^{5}$ Department of Morphology, Surgery and Experimental Medicine, Section of Orthopaedic Clinic, University of Ferrara, \\ Via Aldo Moro 8, Cona, 44124 Ferrara, Italy
}

Correspondence should be addressed to Carlo Cervellati; crvcrl@unife.it

Received 5 March 2015; Revised 12 June 2015; Accepted 21 June 2015

Academic Editor: Marta Ruiz-Ortega

Copyright (C) 2016 Carlo Cervellati et al. This is an open access article distributed under the Creative Commons Attribution License, which permits unrestricted use, distribution, and reproduction in any medium, provided the original work is properly cited.

\begin{abstract}
Postmenopausal osteoporosis (PO) is a major public health issue which affects a large fraction of elderly women. Emerging in vitro evidence suggests a central role of oxidative stress $(\mathrm{OxS})$ in postmenopausal osteoporosis (PO) development. Contrariwise, the human studies on this topic are still scarce and inconclusive. In the attempt to address this issue, we sought to determine if OxS, as assessed by 8 -hydroxy-2-deoxyguanosine $(8-\mathrm{OHdG})$, may influence the level of receptor activator of nuclear factor- $\kappa \mathrm{b}$ ligand (RANKL)/osteoprotegerin (OPG) ratio (a central regulator of bone metabolism) in a sample $(n=124)$, including postmenopausal women with osteoporosis, osteopenia and normal bone mass density (BMD). The most striking result that emerged in our study was the independent and positive (beta $=0.449, p=0.004$, and $R^{2}=0.185$ ) association between the OxS marker and RANKL/OPG ratio which was found in osteopenic but not in the other 2 sample groups. If confirmed by longitudinal studies, our findings would suggest that $\mathrm{OxS}$ is implicated in the derangement of bone homeostasis which precedes PO development. In line with these considerations, antioxidant treatment of postmenopausal women with moderately low BMD might contribute to preventing PO and related complications.
\end{abstract}

\section{Introduction}

Postmenopausal osteoporosis (PO) is a disease characterized by gradual thickening of bone which leads to a reduced bone mass and an increased risk of fragility fractures [1]. PO occurs mostly because of the decline of oestrogens (especially $17 \beta$ estradiol, E2) levels produced by cessation of ovarian sex steroid secretion [2]. This endocrine change has major effects on bone remodelling, leading to derangement of the balance between resorption and formation activities of osteoclasts and osteoblasts, respectively [3]. A vast body of evidence suggests that the effects of E2 on bone are mediated by the mutual interaction of receptor activator of nuclear factor- $\kappa \mathrm{b}$ (RANK), its ligand (RANKL), and osteoprotegerin (OPG) [4-6].

RANKL exists in both soluble and membrane-bound forms and is expressed by many cell types in bone and bone marrow, including osteoblasts, osteocytes, and activated 
lymphocytes [7]. Both forms of this protein promote, although with different effectiveness, bone resorption by binding to RANK localized in both precursors and mature osteoclasts, inducing their formation and activation [8]. Osteoblasts are also one of the main sources of OPG which, acting as a decoy receptor that competes with RANKL for RANK, is able to inhibit osteoclastic proliferation and differentiation. The key-role of RANK/RANKL/OPG axis in the pathogenesis of PO has been largely confirmed in preclinical as well as clinical studies which showed that an increase in RANKL-to-OPG ratio can stimulate excessive bone resorption, whereas its decrease can favor bone neoformation $[5,8,9]$.

Given the centrality of RANK/RANKL/OPG system in bone metabolism, the systemic factors able to regulate the concentration of these cytokines have acquired great scientific and clinical relevance in recent years. Besides E2 (and calciotropic hormones), there are also a series of inflammatory interleukins (e.g., IL-1 and IL-6) that can alter both RANKL and OPG secretion and activity $[5,6]$. Notably, it is now well recognized that the events characterized by burst of these interleukins (i.e., inflammation), but also by physiological decline of E2 (i.e., menopause), are associated with systemic oxidative stress $(\mathrm{OxS})$ [10-12]. This condition can potentially cause the damage against all types of biological molecules and is widely believed to be deeply implicated in the onset and progression of aging-related diseases, including PO [13-16]. More specifically, OxS seems to be a prodromic feature of $\mathrm{PO}$, as suggested by several lines of evidence showing that E2-withdrawal might weaken bone defense against injury induced by reactive oxygen species (ROS) $[15,17,18]$. ROS are, indeed, generated in activated osteoclasts via nicotinamide adenine dinucleotide phosphate oxidase (NOX) and are thought to actively contribute to bone homeostasis "short-circuit" leading to osteoporotic damage [19].

Given these considerations, it was tempting to hypothesize that OxS could play a role in the modulation of RANKL/RANK/OPG triad. On these bases, the aim of the present population-based study was to investigate the potential association between systemic $\mathrm{OxS}$, as assessed by a reliable marker of oxidative damage (urinary 8-hydroxy-2' deoxyguanosine, 8-OHdG), and serum level of RANKL/OPG ratio in a population sample including healthy, osteopenic, and osteoporotic postmenopausal women.

\section{Materials and Methods}

2.1. Subjects. The subjects examined in this study were enrolled among women undergoing bone densitometry evaluation at the Menopause and Osteoporosis Centre of University of Ferrara (Ferrara, Italy), as described elsewhere [20]. The present population-based study was conducted in accordance with the Declaration of Helsinki (World Medical Association, http://www.wma.net) and it was approved by the human research ethics committee of the university. The women were included in the study sample if they were in postmenopausal status, defined as amenorrhea for at least 1 year [21].
Exclusion criteria were use of exogenous sexual hormones (including vaginal estrogens), supplementation with nutritional antioxidants (such as vitamins E, C, and A, betacarotene, and selenium), vegetarian and vegan diet, chronic diseases (such as diabetes, malabsorption, and cardiovascular disease), or being not diagnosed with a chronic disease, but taking medications (antiobesity medications, thyroid hormones, diuretics, antihypertensive, anticholesterol drugs, etc.).

One hundred twenty-four subjects were found to be eligible and were enrolled in the study after signing an informed consent. Body weight, height and waist circumference were assessed in each enrolled subjects by trained personnel.

2.2. Biochemical Assays. Fresh blood samples were obtained from antecubital vein from all subjects between 8.30 and $10.00 \mathrm{am}$, after fasting for at least $8 \mathrm{~h}$. After 30 minutes of incubation at room temperature (RT), blood samples were centrifuged $(3000 \mathrm{~g}$ for $10 \mathrm{~min})$, and the obtained serum was stored at $-80^{\circ} \mathrm{C}$ until analysis. Commercially available Enzyme-Linked Immunosorbent Assays (ELISAs) kits were performed, according to the manufacturer's instructions.

Serum level of total (free plus bound) soluble RANKL was assayed by Human sRANKL (total) ELISA (catalog number RD193004200R, purchased from BioVendor Research and Diagnostic Products, Modrice, Czech Republic). In brief, standards, quality controls, or samples (100 $\mu \mathrm{L}$ each) were incubated in microplate wells precoated with monoclonal anti-human sRANKL antibody. After a 16-20-hour incubation (at $2-8^{\circ} \mathrm{C}$ ), the plate was washed and incubated for 60 minutes at RT with biotin labelled polyclonal anti-human sRANKL antibody. After a further washing step, streptavidinHRP conjugate was added and incubated for 60 minutes (RT). Then the plate was rewashed and the remaining conjugate was allowed to react with the substrate solution containing hydrogen peroxide and tetramethylbenzidine (TMB). After stopping the reaction, the plate was read at $450 \mathrm{~nm}$. The concentration of RANKL in the serum samples was estimated from the standard curve and expressed as pmol/L (detection limit: $0.4 \mathrm{pmol} / \mathrm{L}$ ). The intra-assay $\mathrm{CV}$ was $9.3 \%$, whereas the interassay CV was $11.0 \%$.

Serum concentration of OPG was detected by OPG ELISA kit (catalog number EK0480, Boster Biological Technology Co., Ltd., China). One hundred $\mu \mathrm{L}$ of either standards or properly diluted serum samples was added into antihuman OPG antibody precoated wells. After incubation (90 minutes at $37^{\circ} \mathrm{C}$ ), biotinylated anti-human OPG antibody was added into each well and the plate was reincubated at the same temperature for a shorter time interval (60 minutes). Following the first washing step, a solution containing avidin, biotin, and peroxidase was added into each well of the plate which was then incubated for further 30 minutes $\left(37^{\circ} \mathrm{C}\right)$ and thoroughly washed one more time. Afterwards, TMB color developing agent was added and, eventually, the absorbance was read at $450 \mathrm{~nm}$. The serum concentration of OPG was estimated from the standard curve and expressed as $\mathrm{pmol} / \mathrm{L}$ (detection limit: $1 \mathrm{pmol} / \mathrm{L})$. The intra-assay $\mathrm{CV}$ was $5.3 \%$, whereas the interassay $\mathrm{CV}$ was $7.0 \%$. 
Serum concentration of bone-specific alkaline phosphatase (BAP) was detected by OCTEIA Ostase BAP immunoenzymometric assay (catalog number AC-20F1, purchased by Immunodiagnostic Systems Ltd., Boldon, UK). Fifty $\mu \mathrm{L}$ of either standards, controls, or serum specimens was pipetted into streptavidin precoated wells and subsequently mixed with a biotin-labelled BAP-specific monoclonal antibody. After incubation (1 hour at RT), substrate reagent solution (i.e., p-nitrophenyl phosphate) was added into each well. A further incubation (15 minutes at RT) was followed by the addition of stop solution into each well. The absorbance was finally read at $405 \mathrm{~nm}$ (subtracting blank reading at $650 \mathrm{~nm}$ ). The serum concentration of BAP was estimated from the standard curve and expressed as $\mu \mathrm{g} / \mathrm{L}$ (detection limit: $0.7 \mu \mathrm{g} / \mathrm{L}$ ). The intra-assay $\mathrm{CV}$ was $4.1 \%$, whereas the interassay CV was $5.5 \%$.

Serum concentration of C-terminal telopeptides of Type I (CTX-1) was measured by serum Cross-Laps ELISA kit (catalog number AC-02F1, purchased by Immunodiagnostic Systems Ltd., Boldon, UK). Briefly, $50 \mu \mathrm{L}$ of either standards, control, or serum samples was pipetted into streptavidin precoated wells followed by the addition of the antibody solution (containing biotinylated monoclonal murine antibody plus monoclonal murine antibody conjugated with peroxidase). After 2 hours of incubation at RT, wells were washed and then chromogenic substrate (TMB) solution was added. Measurement of the absorbance at $450 \mathrm{~nm}$ with $650 \mathrm{~nm}$ as reference was made within two hours after the addition of the stop solution. The concentration of CTX-1 in the serum samples was obtained by standard curve and was expressed as $\mathrm{ng} / \mathrm{mL}$ (detection limit: $0.020 \mathrm{ng} / \mathrm{mL}$ ). The intra-assay CV was $2.2 \%$, whereas the interassay CV was $7.7 \%$.

High sensitivity C-reactive protein (Hs-CRP) serum concentration was assessed by commercial kit Hs-CRP the EiAsyTM Way (catalog number CAN-CRP-4360, purchased from Diagnostics Biochem Canada Inc., Dorchester, CAN). Twenty $\mu \mathrm{L}$ of either each calibrator, control, or properly diluted serum sample was pipetted into mouse anti-CRP precoated wells. After 30 minutes of incubation (RT) followed by a washing step, anti-CRP monoclonal conjugated with horseradish peroxidase was added into each well. After 15 minutes at RT, followed by a washing step, TMB substrate solution was added and, eventually, the absorbance was read at $450 \mathrm{~nm}$ within 20 minutes after the addition of the stop solution. The concentration of Hs-CRP in the serum samples was estimated from the standard curve and expressed as $\mathrm{ng} / \mathrm{mL}$ (detection limit: $10 \mathrm{ng} / \mathrm{mL}$ ). The intra-assay CV was $9.5 \%$, whereas the interassay $\mathrm{CV}$ was $9 \%$.

Urine concentration of 8-OHdG was detected by competitive 8-OHdG EIA kit (catalog number SKT-120-96, purchased from StressMarq Biosciences Inc., Victoria, BC, Canada). Fifty $\mu \mathrm{L}$ of either standard or properly diluted urine specimens was added into wells precoated with goat antimouse IgG. Afterwards, 2 equal volume aliquots of 8-OHdGacetylcholinesterase conjugate and 8-OHdG monoclonal antibody were added to each well. After an overnight incubation $\left(4^{\circ} \mathrm{C}\right)$ Ellman's reagent [5, $5^{\prime}$-dithiobis-(2-nitrobenzoic acid)] was added to each well. The absorbance was finally read at $405 \mathrm{~nm}$. The concentration of $8-\mathrm{OHdG}$ in the urine samples was obtained by standard curve and was expressed as $\mathrm{ng} / \mathrm{mL}$ (detection limit: $0.033 \mathrm{ng} / \mathrm{mL}$ ). The intra-assay CV was $7.8 \%$, whereas the interassay CV was $6.4 \%$. The 8 OHdG concentration was normalized to urinary creatinine concentration and expressed as $\mathrm{ng} / \mathrm{mg}$ creatinine.

Urinary creatinine determination was performed by a picric acid method [22]. Briefly, $50 \mu \mathrm{L}$ of either standard or properly dilute urine samples was added into microplate well and mixed with $200 \mu \mathrm{L}$ of a working solution containing $25 \mathrm{mM}$ picric acid (purchased form Sigma-Aldrich, St. Louis, $\mathrm{MO}, \mathrm{USA}$ ) and $130 \mathrm{mM} \mathrm{NaOH}$. The $490 \mathrm{~nm}$ absorbance was read after 30 minutes of incubation at RT and the obtained concentration was expressed as $\mathrm{mg} / \mathrm{dL}$ (detection limit: $0.1 \mathrm{mg} / \mathrm{dL}$ ). The intra-assay CV was $5.3 \%$, whereas the interassay $\mathrm{CV}$ was $7.6 \%$.

All the above ELISAs were assayed by a Tecan infinite (M200 Tecan Group Ltd., Männedorf, Switzerland) microplate spectrophotometer.

2.3. Bone Densitometry Assessment. Areal bone density was assessed at lumbar spine, hip, and total body by Discovery dual energy X-ray absorptiometry scanner (Hologic Inc., Bedford, MA). PO was diagnosed when BMD T-score (the number of standard deviations below the average for a young adult at peak bone density) was lower than 2.5 standard deviations from BMD peak at either femoral neck or lumbar spine, according to WHO guidelines [23]. In accordance with these criteria, women with $T$-score at either skeleton area between -2.5 and -1.0 were classified as osteopenic and those with a value higher than -1.0 as normal.

2.4. Statistical Analysis. SPSS 18.0 for Windows (IBM, Chicago, IL, USA) was used for statistical analysis. All variables were first analyzed for the normal distribution by the Kolmogorov-Smirnov and the Shapiro-Wilkinson test. Differences between groups were checked by one way analysis of variance (ANOVA) and Kruskal-Wallis for normally and non-normally distributed variables, respectively. Univariate analysis (by Pearson's or Spearman's test, depending on the distribution of the variable) was performed to check the associations between selected variables. Simple and multiple linear regression analysis were performed using base-10 logarithm transformed values of RANKL, OPG, RANKL/OPG, and 8-OHdG. We used log-transformed variables for these analyses to meet the assumption of normality of regression residuals. A two-tailed probability value $<0.05$ was considered statistically significant.

\section{Results}

The main characteristics of the 124 postmenopausal women enrolled in the present study are shown in Table 1. Osteoporotic and osteopenic women were older $(p=0.009)$ and presented lower BMI $(p=0.04)$ and waist circumference $(p=0.03)$ compared to those with BMD values within normal range. In accordance with the diagnostic criteria, total hip, neck, and lumbar spine BMD, as well as the correspondent $T$-score values, were significantly $(p<0.01)$ 
TABLE 1: Principal characteristics of normal, osteopenic, and osteoporotic postmenopausal women.

\begin{tabular}{|c|c|c|c|c|}
\hline & $\begin{array}{l}\text { Normal BMD } \\
\quad(n=25)\end{array}$ & $\begin{array}{c}\text { Osteopenia } \\
(n=59)\end{array}$ & $\begin{array}{l}\text { Osteoporosis } \\
\quad(n=40)\end{array}$ & $\begin{array}{c}\text { Statistics } \\
p \\
\end{array}$ \\
\hline Age, yr & $54.0 \pm 4.2$ & $56.2 \pm 4.5$ & $57.7 \pm 4.7$ & $0.01^{*}$ \\
\hline Years since menopause, yr & $3(1-9)$ & $5(2-10)$ & $7(4-12)$ & $0.09^{\#}$ \\
\hline BMI, $\mathrm{kg} / \mathrm{m}^{2}$ & $24.2(23.0-27.4)$ & $23.1(21.5-27.3)$ & $23.2(22.1-26.7)$ & $0.16^{\#}$ \\
\hline Waist circumference, $\mathrm{cm}$ & $85.9 \pm 10.9$ & $83.2 \pm 8.4$ & $82.5 \pm 8.9$ & $0.05^{*}$ \\
\hline \multicolumn{5}{|l|}{ DXA parameters } \\
\hline L. spine $\mathrm{BMD}, \mathrm{g} / \mathrm{cm}^{2}$ & $1.10 \pm 0.91$ & $0.92 \pm 0.11$ & $0.83 \pm 0.09$ & $<0.01^{*}$ \\
\hline L. spine $T$-score & $0.1(-0.6$ to 0.3$)$ & $-1.8(-2.1$ to -1.2$)$ & $-2.5(-2.8$ to -1.8$)$ & $<0.01^{\#}$ \\
\hline F. neck BMD, $\mathrm{g} / \mathrm{cm}^{2}$ & $0.81(0.79-0.86)$ & $0.67(0.63-0.71)$ & $0.62(0.57-0.64)$ & $<0.01^{\#}$ \\
\hline F. neck $T$-score & $-0.3(-0.6$ to 0.0$)$ & $-1.6(-2.0$ to -1.2$)$ & $-2.1(-2.5$ to -1.8$)$ & $<0.01^{\#}$ \\
\hline Total hip BMD, $\mathrm{g} / \mathrm{cm}^{2}$ & $0.93 \pm 0.08$ & $0.81 \pm 0.07$ & $0.74 \pm 0.07$ & $<0.01^{*}$ \\
\hline Total hip $T$-score & $-0.1(-0.4$ to 0.4$)$ & $-1.1(-1.5$ to -0.8$)$ & $-1.5(-2.0$ to -1.2$)$ & $<0.01^{\#}$ \\
\hline \multicolumn{5}{|l|}{ Biochemical markers } \\
\hline Hs-CRP, mg/L & $1.5(0.6-3.7)$ & $1.1(0.6-2.1)$ & $1.2(0.4-2.6)$ & 0.54 \\
\hline CTX-1, ng/mL & $0.47 \pm 0.21$ & $0.46 \pm 0.39$ & $0.52 \pm 0.30$ & $0.47^{*}$ \\
\hline BAP, $\mu \mathrm{g} / \mathrm{L}$ & $30.3 \pm 1.3$ & $31.0 \pm 1.0$ & $25.1 \pm 1.3$ & $0.24^{*}$ \\
\hline RANKL, pmol/L & $270(201-362)$ & $255(157-347)$ & $281(166-365)$ & $0.18^{\#}$ \\
\hline OPG, pmol/L & $8.1(7.3-10.6)$ & $11.0(6.9-16.6)$ & $12.0(5.7-18.6)$ & $0.78^{\#}$ \\
\hline RANKL/OPG & $31.0(18.2-64.1)$ & $20.1(11.5-60.1)$ & $21.2(11.4-34.2)$ & $0.31^{\#}$ \\
\hline 8-OHdG, ng/mg creatinine & $143(109-189)$ & $154(109-208)$ & $159(112-212)$ & $0.35^{\#}$ \\
\hline
\end{tabular}

Data presented are expressed as mean \pm standard deviation for normally distributed variables; median (interquartile range) for not normally distributed variables.

* $p$ value by Kruskal-Wallis; ${ }^{*} p$ value by ANOVA.

BMI: body mass index; BMD: bone mass density; L.: lumbar; F.: femoral; Hs-CRP: high reactivity C-reactive protein; CTX-1: C-terminal telopeptide of type I collagen; BAP: bone-specific alkaline phosphatase; OPG: osteoprotegerin; RANKL: receptor activator of nuclear factor kappa-B ligand; 8-OHdG: 8-hydroxy$2^{\prime}$-deoxyguanosine.

higher in controls with respect to osteopenic and osteoporotic women. In contrast, serum level of Hs-CRP, RANKL, OPG, RANKL/OPG ratio, CTX-1, BAP, and urinary level of 8OHdG did not significantly vary among the three sample groups.

The possible association of 8-OHdG with the other biochemical markers and BMD values was initially checked by simple correlation analysis (Table 2). From this test it emerged that the DNA damage marker was significantly correlated only with RANKL ( $p=0.003)$ and RANKL/OPG ratio $(p=0.002)$.

Afterwards, we checked the association between 8-OHdG and the two cytokines within each sample group (Table 3). As displayed in the table and in Figure 1, the OxS marker resulted to be significantly and positively correlated with RANKL ( $p=$ $0.005)$ and RANK/OPG ( $p=0.004)$ merely in the osteopenic group, with a percentage of variance explained equal to 18.0 and $18.2 \%$, respectively. Of note, the linear standardized coefficient for the association between 8-OHdG and OPG was negative (beta $=-0.196$ ) and, although not statistically significant ( $p=0.098)$, markedly higher than those obtained among controls (beta $=-0.037$ ) and osteoporotic (beta $=$ $-0.089)$ women.

Finally, in order to unveil if the correlations found to be significant in the osteopenic group were independent of potential confounding factors, we performed two multiple
TABLE 2: Simple correlation between 8-OHdG and RANKL, OPG and RANKL/OPG, BMD values, and bone resorption/formation markers (total sample, $n=124$ ).

\begin{tabular}{lc}
\hline & 8 -OHdG \\
\hline L. spine BMD & -0.13 \\
F. neck BMD & 0.04 \\
Total hip BMD & 0.03 \\
CTX-1 & 0.07 \\
BAP & 0.06 \\
RANKL & $0.263^{*}$ \\
OPG & -0.116 \\
RANKL/OPG & $0.277^{*}$ \\
\hline
\end{tabular}

${ }^{*} p<0.01$ by Pearson's analysis of base- 10 logarithm transformed values of the 2 variables.

BMI: body mass index; BMD: bone mass density; L.: lumbar; F.: femoral; CTX-1: C-terminal telopeptide of type I collagen; BAP: bone-specific alkaline phosphatase; OPG: osteoprotegerin; RANKL: receptor activator of nuclear factor kappa-B ligand; 8-OHdG: 8-hydroxy-2' ${ }^{\prime}$-deoxyguanosine.

regression models including age, years since menopause, BMI, waist circumference, and Hs-CRP as covariates (Table 4). These analyses showed that both associations, 8-OHdG versus RANKL and 8-OHdG versus RANKL/OPG, retained their significance even after multiple adjustments. 
TABLE 3: Simple linear regression analysis for the relationship between urinary level of 8-OHdG and serum levels of RANKL, OPG, and RANKL/OPG ratio in normal, osteopenic, and osteoporotic postmenopausal women.

\begin{tabular}{|c|c|c|c|c|}
\hline & & Normal BMD & Osteopenia & Osteoporosis \\
\hline \multirow{3}{*}{ RANKL } & $B$ (DS) & $0.325(0.389)$ & $0.554(0.164)$ & $0.018(0.212)$ \\
\hline & Beta & 0.188 & $0.423^{*}$ & 0.014 \\
\hline & $R^{2}$ & 0.035 & 0.180 & 0.001 \\
\hline \multirow{3}{*}{ OPG } & $B$ (DS) & $-0.048(0.288)$ & $-0.256(0.175)$ & $-0.093(0.256)$ \\
\hline & Beta & -0.037 & -0.196 & -0.089 \\
\hline & $R^{2}$ & 0.001 & 0.038 & 0.004 \\
\hline \multirow{3}{*}{ RANKL/OPG } & $B$ (DS) & $0.368(0.549)$ & $0.879(0.262)$ & $0.161(315)$ \\
\hline & Beta & 0.160 & $0.429^{*}$ & 0.086 \\
\hline & $R^{2}$ & 0.026 & 0.184 & 0.007 \\
\hline
\end{tabular}

${ }^{*} p<0.001$.

Beta: standardized regression coefficient; $B$ : nonstandardized regression coefficient; BMD: bone mass density; OPG: osteoprotegerin; RANKL: receptor activator of nuclear factor kappa-B ligand; 8-OHdG: 8-hydroxy-2' ${ }^{\prime}$-deoxyguanosine.

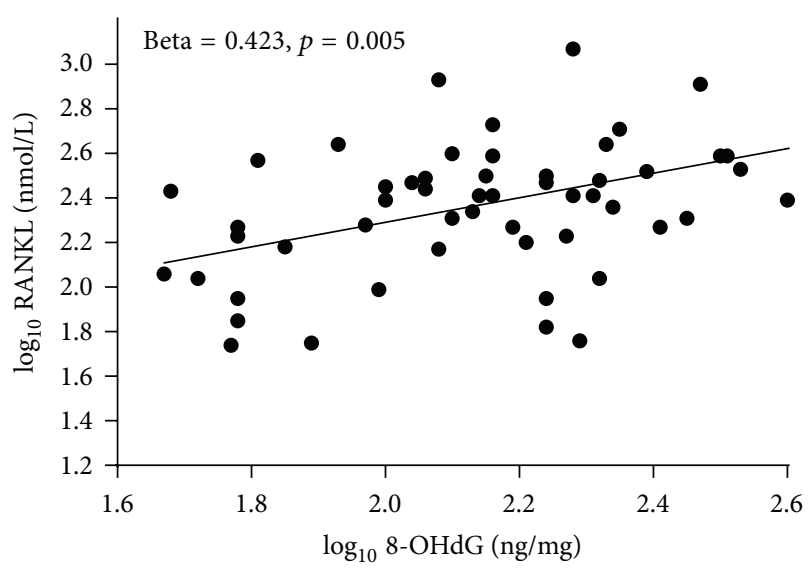

(a)

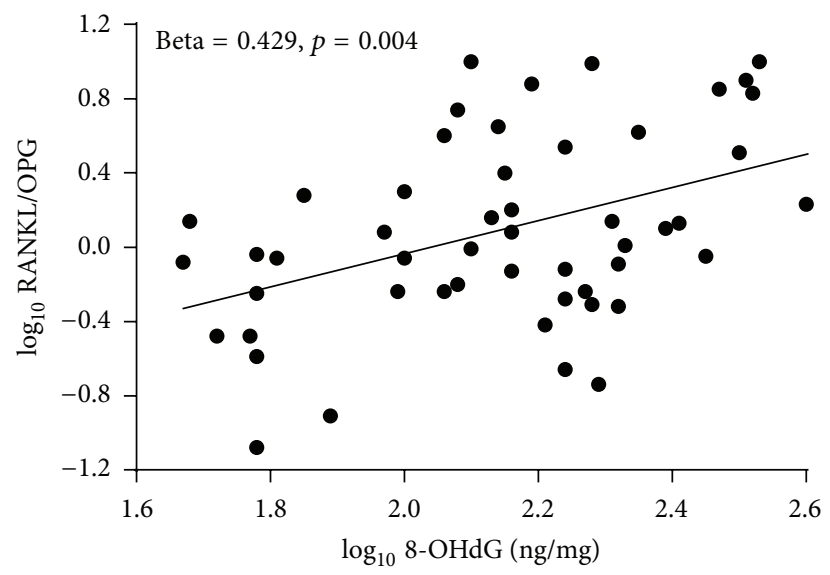

(b)

FIGURE 1: Box plots of the correlations: $\log _{10} 8$-OHdG versus $\log _{10}$ RANKL (right); $\log _{10} 8$-OHdG versus $\log _{10}$ RANKL/OPG.

\section{Discussion}

In the present study, we evaluated the possible link between $\mathrm{PO}$ and $\mathrm{OxS}$, as assessed by $8-\mathrm{OHdG}$, by various angles. The analyses of the data obtained revealed that this OxS marker was not significantly associated with diagnosis of osteopenia/osteoporosis; $\mathrm{BMD}$ of different skeleton area; resorption/formation bone markers. In contrast, we found that higher level of 8-OHdG was strongly and independently related to increased serum concentration of RANKL and RANKL/OPG among postmenopausal women with osteopenia but not among those with normal BMD or osteoporosis.

In line with our results, several previous works [15, $16,24,25]$ did not find any significant difference in the peripheral level of $\mathrm{OxS}$ between osteoporotic and healthy postmenopausal women. In contrast, some of these studies $[15,16]$, along with others $[14,26,27]$, found an inverse, although statistically weak, correlation between $\mathrm{OxS}$ and femoral neck and/or lumbar spine BMD. In our point of view, the reasons of these discrepancies mostly lay in the different indicators employed for peripheral OxS determination. Indeed, almost all the aforementioned studies dealt with markers (such as malondialdehyde, hydroperoxides, and F2isoprostanes) which are all derived by lipid peroxidation. This cascade reaction is markedly different from the DNA repair process yielding $8-\mathrm{OHdG}$. Differently from the former, lipoperoxidation leads to the formation of several byproducts, which are very reactive and can markedly amplify the initial ROS-induced oxidative spark. Furthermore, DNA oxidative injury merely occurs inside the cells, whereas the targets of lipoperoxidation are disseminated both in and out of the cytosol (e.g., membranes of cell and organelles and lipid moiety of circulating lipoproteins). Consistent with these observations, the precious systematic work by Kadiiska and colleagues suggests that there could be different forms of OxS, and each might bring about the rise of a different series of peripheral markers [28].

Our finding of the absence of a detectable increase in systemic $\mathrm{OxS}$ in relation to $\mathrm{PO}$ occurrence does not rule out that reactive species can play a role in the development of this bone disease. The gathered results also showed, indeed, that $\mathrm{OxS}$ might be an effective influencing factor of RANK/RANKL/OPG triad, which plays a paramount role in the pathogenesis of $\mathrm{PO}$ and other metabolic bone 
TABLE 4: Multiple regression analysis for the relationship between 8-OHdG and RANKL and RANKL/OPG, among osteopenic postmenopausal women $(n=59)$.

\begin{tabular}{lcccc}
\hline \multirow{2}{*}{ Predictors } & \multicolumn{2}{c}{$\begin{array}{c}\text { Dependent variable } \\
\text { RANKL }\end{array}$} & \multicolumn{2}{c}{$\begin{array}{c}\text { Dependent variable } \\
\text { RANKL/OPG }\end{array}$} \\
& Beta & $p$ value & Beta & $p$ value \\
\hline 8-OHdG & 0.469 & 0.002 & 0.449 & 0.004 \\
Age & 0.071 & 0.694 & 0.119 & 0.644 \\
Years since menopause & 0.031 & 0.849 & 0.015 & 0.930 \\
BMI & 0.016 & 0.939 & 0.059 & 0.274 \\
Waist circumference & 0.001 & 0.998 & -0.049 & 0.812 \\
Hs-CRP & -0.115 & 0.411 & -0.079 & 0.523 \\
\hline & \multicolumn{2}{c}{$R^{2}=0.202$} & \multicolumn{2}{c}{$R^{2}=0.185$}
\end{tabular}

Beta: standardized regression coefficient; BMI: body mass index; BMD: bone mass density; Hs-CRP: high reactivity C-reactive protein; RANKL: receptor activator of nuclear factor kappa-B ligand; 8-OHdG: 8-hydroxy-2' deoxyguanosine.

diseases $[5,6,29,30]$. This discovery adds to the current literature, because, to the best of our knowledge, it is the first time that such interaction is found in human subjects. Conversely, there is abundant supportive evidence from in vitro experiments on various cell lineages such as mouse osteoblasts, human MG63, and primary bone marrow cell cultures [14, 31]. More in detail, Baek and coworkers showed that oxygen peroxide can promote the number and activity of osteoclasts and RANKL expression, but not OPG. Of interest, these effects were abolished upon adding catalase, a potent $\mathrm{H}_{2} \mathrm{O}_{2}$-scavenger [14]. Increase in endogen ROS burden leading to enhancement of RANKL production of osteoclast precursor cells can be derived by NOX activation or by a reduced expression of nuclear factor (erythroidderived 2-) like 2 (Nrf2), as shown in a recent work on Nrf2 knockout mice [13]. Noteworthily, the partial activation of this redox-sensitive transcription factor, which regulates the expression of several genes encoding essential antioxidant enzymes, resulted in inhibition of osteoclast differentiation [13].

The clinical importance of the present study derives from the fact that RANK/RANKL/OPG axis is now widely regarded as one of the most promising molecular targets for novel therapeutic approaches in the management of bone diseases $[4,30]$. Accordingly, the inhibition of RANKL by denosumab (a fully human antibody against RANKL) was more effective at reducing the occurrence of vertebral fractures than the traditional drugs [9]. In spite of these encouraging outcomes, there is still an intense demand for alternative, nonpharmaceutical (and, at least hopefully, safer) interventions on this high-incidence disease. In this context the in vitro and animal data highlighting the protective effects on bone elicited by various antioxidants such as lycopene [32], resveratrol [33], and tocotrienol [34] are promising.

Unfortunately, the human observational studies set out to examine the effects of antioxidants on bone health are still sparse and controversial [35] and do not allow translating the preclinical evidence in an effective antiosteoporotic treatment. Moreover, the interpretation of the epidemiological is difficult because most of these studies are affected by important limitations such as cross-sectional design [24, 36, 37] and lack of measurement of circulatory antioxidants concentration which should accompany the evaluation of nutrients intake by dedicated questionnaires [24,38]. The latter point is of primary importance, because the bioavailability of these compounds depends on the food matrix consumed and on genetic variability and physiological condition of the subjects [35]. Besides, the findings may be biased by the interference of other nutrients as suggested by the authors of one of the few longitudinal studies on this field [38]. Indeed, examining a sample of 891 women, Macdonald et al. observed significant negative correlations between BMD and nutrients, in particular vitamin $\mathrm{E}$ (from diet alone) and polyunsaturated fatty acids (PUFAs). The researchers suggest that the strong association that was also found between PUFAs and vitamin E intake $(r=0.822, p<0.001)$ could account for this unexpected result and, as consequence, this vitamin could simply represent a surrogate marker for fat intake [38]. Disappointing data were also obtained in a crosssectional study by Wolf et al. [36], where dietary and total intake, or serum concentration of vitamin $\mathrm{E}, \beta$-carotene, lycopene, and other antioxidants, failed to be associated with BMD in women ( $n=11068$, aged 50-79 years). Contrariwise, a clear beneficial effect of $\alpha$-tocopherol was found in a recent longitudinal study which showed that low intake and serum concentration of the vitamin were both associated with an increased rate of bone fracture in both elderly women $(n=61422)$ and men $(n=1138)$ [39]. Finally, similar bone protective effects of carotenoids [40] or vitamin C [41] emerged from data collected in Framingham Osteoporosis Study.

Overall, the published epidemiological studies, although presenting some controversies and design issues, appear to support the commonly held belief that antioxidant-rich fruits improve bone health and are strongly suggestive of a beneficial role of these bioactive molecules [31]. However, one must be aware that it is not yet completely clear if these osteoprotective effects are merely exerted by an antioxidant pathway or by the simple restoring of mineral balance and/or vitamin K bioavailability [35, 36].

Thus, well-designed, randomized, controlled studies are warranted to confirm the findings from the animal studies on bone loss and subsequent development of osteoporosis. In our view, however, the concept that has to be borne in mind is the following: PO is a multifactor and multifaceted disease, and, thus, OxS should not be considered as the unique enemy to defeat. Owing to these considerations, it is conceivable to assume that antioxidants alone could not represent the definitive treatment for PO but more likely as therapeutic adjuvant of well-established antiosteoporotic drugs. The indication that emerged from our investigation was that this type of supplements might benefit postmenopausal women with osteopenia. Indeed only in those subjects, where bone remodeling cycle is altered, but not still completely compromised, the RANK/RANK/OPG system appeared to be sensitive to the elevation of 8-OHdG. Therefore, OxS, during this condition defined as prelude of PO, could contribute to uncoupling the balance between bone resorption and 
formation, thus guiding the process of bone degeneration to the "definitive" osteoporotic damage. Arresting this process, before BMD is not too low $(T$-score $<2.5)$, is very important to prevent from onset of $\mathrm{PO}$ and related fragility fractures [31].

Finally, some important limitations of the study must be acknowledged. First, the design of the study was crosssectional, thereby precluding our ability to establish any temporal relationship between the markers examined. Therefore, longitudinal investigations are mandatory to draw a definitive appreciation of causal nature of $\mathrm{OxS}$ with respect to alteration in circulatory level of RANKL and RANKL/OPG. Second, the lack of a full nutritional assessment of the sample subjects makes it difficult to rule out the fact that dietary antioxidant intake might interfere with the assessed level of 8-OHdG and, hence, with the reliability of study outcomes. However, to attenuate the influence of this factor, all the individuals reporting the use of antioxidant supplements or to follow a vegetarian diet were excluded a priori from the study. Third, the serum level of RANKL and OPG might not reflect the levels and activity of these cytokines in bone microenvironment, and a portion of them could originate from nonskeletal sources, in particular inflammation $[4,5]$. In this regard, in the attempt to limit the potential interference of this factor on our statistical outcomes, we included Hs-CRP in the multivariate analysis. Finally, a limitation of the fact that RANKL/OPG is not a suitable marker for PO diagnosis could appear. On the contrary, it may represent a potential strength of the mounting consensus around the use of this peripheral index for monitoring bone health and antiosteoporotic therapy response of patients with bone diseases [42].

We would like to also underline some other strengths of the present work. To the best of our knowledge, this is the first study that provides in vivo data in support of the interaction between RANKL/OPG and OxS among women with high risk of osteoporosis. Noteworthily this correlation resulted to be independent by potential confounders such as age and measures of body fat. Moreover, we consider as further study strength the use of a widely recognized reliable marker of DNA oxidative damage such as urinary $8-\mathrm{OHdG}[28,43]$.

\section{Conclusion}

In conclusion, our findings demonstrate the existence of a positive association between systemic $\mathrm{OxS}$ and serum level RANKL/OPG ratio in osteopenic but not in normal and osteoporotic postmenopausal women. Thus, the data obtained, although warranted confirmation by longitudinal studies, suggest that women with moderately low BMD could be the target population for antioxidant-based interventions aimed at preventing osteoporosis-related bone loss and fracture.

\section{Conflict of Interests}

The authors declare that there is no conflict of interests regarding the publication of this paper.

\section{Acknowledgments}

The authors thank Cristina Castaldini and Stefania Ferrazzini for their skilled organization of clinical protocol. They also want to thank Manuela Spagnolo and Ilaria Crivellari for their meaningful contributions in data collection and processing. The study was supported by "Local Research Project" Grant from University of Ferrara, Italy.

\section{References}

[1] L. Masi and M. L. Brandi, "Physiopathological basis of bone turnover," Quarterly Journal of Nuclear Medicine, vol. 45, no. 1, pp. 2-6, 2001.

[2] M. N. Weitzmann and R. Pacifici, "Estrogen deficiency and bone loss: an inflammatory tale," The Journal of Clinical Investigation, vol. 116, no. 5, pp. 1186-1194, 2006.

[3] L. G. Raisz, "Pathogenesis of osteoporosis: concepts, conflicts, and prospects," Journal of Clinical Investigation, vol. 115, no. 12, pp. 3318-3325, 2005.

[4] A. E. Kearns, S. Khosla, and P. J. Kostenuik, "Receptor activator of nuclear factor $\kappa \mathrm{B}$ ligand and osteoprotegerin regulation of bone remodeling in health and disease," Endocrine Reviews, vol. 29, no. 2, pp. 155-192, 2008.

[5] D. Vega, N. M. Maalouf, and K. Sakhaee, "The role of receptor activator of nuclear factor- $\kappa \mathrm{B}$ (RANK)/RANK ligand/osteoprotegerin: clinical implications," Journal of Clinical Endocrinology and Metabolism, vol. 92, no. 12, pp. 4514-4521, 2007.

[6] S. Bord, D. C. Ireland, S. R. Beavan, and J. E. Compston, “The effects of estrogen on osteoprotegerin, RANKL, and estrogen receptor expression in human osteoblasts," Bone, vol. 32, no. 2, pp. 136-141, 2003.

[7] T. Nakashima, M. Hayashi, T. Fukunaga et al., "Evidence for osteocyte regulation of bone homeostasis through RANKL expression," Nature Medicine, vol. 17, no. 10, pp. 1231-1234, 2011.

[8] S. A. J. Lloyd, Y. Y. Yuan, P. J. Kostenuik et al., "Soluble RANKL induces high bone turnover and decreases bone volume, density, and strength in mice," Calcified Tissue International, vol. 82, no. 5, pp. 361-372, 2008.

[9] N. Freemantle, C. Cooper, A. Diez-Perez et al., "Results of indirect and mixed treatment comparison of fracture efficacy for osteoporosis treatments: a meta-analysis," Osteoporosis International, vol. 24, no. 1, pp. 209-217, 2013.

[10] F. Pansini, C. Cervellati, A. Guariento et al., "Oxidative stress, body fat composition, and endocrine status in pre- and postmenopausal women," Menopause, vol. 15, no. 1, pp. 112-118, 2008.

[11] C. Cervellati, F. S. Pansini, G. Bonaccorsi et al., " $17 \beta$-estradiol levels and oxidative balance in a population of pre-, peri-, and post-menopausal women," Gynecological Endocrinology, vol. 27, no. 12, pp. 1028-1032, 2011.

[12] A. Muñoz and M. Costa, "Nutritionally mediated oxidative stress and inflammation," Oxidative Medicine and Cellular Longevity, vol. 2013, Article ID 610950, 11 pages, 2013.

[13] S. Hyeon, H. Lee, Y. Yang, and W. Jeong, "Nrf2 deficiency induces oxidative stress and promotes RANKL-induced osteoclast differentiation," Free Radical Biology and Medicine, vol. 65, pp. 789-799, 2013.

[14] K. H. Baek, K. W. Oh, W. Y. Lee et al., "Association of oxidative stress with postmenopausal osteoporosis and the effects of 
hydrogen peroxide on osteoclast formation in human bone marrow cell cultures," Calcified Tissue International, vol. 87, no. 3, pp. 226-235, 2010.

[15] C. Cervellati, G. Bonaccorsi, E. Cremonini et al., "Bone mass density selectively correlates with serum markers of oxidative damage in post-menopausal women," Clinical Chemistry and Laboratory Medicine, vol. 51, no. 2, pp. 333-338, 2013.

[16] C. Cervellati, G. Bonaccorsi, E. Cremonini et al., "Oxidative stress and bone resorption interplay as a possible trigger for postmenopausal osteoporosis," BioMed Research International, vol. 2014, Article ID 569563, 8 pages, 2014.

[17] L. Ibáñez, M. L. Ferrándiz, R. Brines, D. Guede, A. Cuadrado, and M. J. Alcaraz, "Effects of Nrf2 deficiency on bone microarchitecture in an experimental model of osteoporosis," Oxidative Medicine and Cellular Longevity, vol. 2014, Article ID 726590, 9 pages, 2014.

[18] J. M. Lean, J. T. Davies, K. Fuller et al., "A crucial role for thiol antioxidants in estrogen-deficiency bone loss," The Journal of Clinical Investigation, vol. 112, no. 6, pp. 915-923, 2003.

[19] A. G. Darden, W. L. Ries, W. C. Wolf, R. M. Rodriguiz, and L. L. Key Jr., "Osteoclastic superoxide production and bone resorption: stimulation and inhibition by modulators of NADPH oxidase," Journal of Bone and Mineral Research, vol. 11, no. 5, pp. 671-675, 1996.

[20] C. Cervellati, F. S. Pansini, G. Bonaccorsi et al., "Body mass index is a major determinant of abdominal fat accumulation in pre-, peri- and post-menopausal women," Gynecological Endocrinology, vol. 25, no. 6, pp. 413-417, 2009.

[21] S. D. Harlow, S. Crawford, L. Dennerstein, H. G. Burger, E. S. Mitchell, and M.-F. Sowers, "Recommendations from a multistudy evaluation of proposed criteria for staging reproductive aging," Climacteric, vol. 10, no. 2, pp. 112-119, 2007.

[22] J. G. H. Cook, "Factors influencing the assay of creatinine," Annals of Clinical Biochemistry, vol. 12, no. 6, pp. 219-232, 1975.

[23] J. A. Kanis, "Osteoporosis III: diagnosis of osteoporosis and assessment of fracture risk," The Lancet, vol. 359, no. 9321, pp. 1929-1936, 2002.

[24] D. Maggio, M. Barabani, M. Pierandrei et al., "Marked decrease in plasma antioxidants in aged osteoporotic women: results of a cross-sectional study," The Journal of Clinical Endocrinology \& Metabolism, vol. 88, no. 4, pp. 1523-1527, 2003.

[25] M. A. Sánchez-Rodríguez, M. Ruiz-Ramos, E. Correa-Muñoz, and V. M. Mendoza-Núñez, "Oxidative stress as a risk factor for osteoporosis in elderly Mexicans as characterized by antioxidant enzymes," BMC Musculoskeletal Disorders, vol. 8, article 124, 2007.

[26] S. Basu, K. Michaëlsson, H. Olofsson, S. Johansson, and H. Melhus, "Association between oxidative stress and bone mineral density," Biochemical and Biophysical Research Communications, vol. 288, no. 1, pp. 275-279, 2001.

[27] O. F. Sendur, Y. Turan, E. Tastaban, and M. Serter, "Antioxidant status in patients with osteoporosis: a controlled study," Joint Bone Spine, vol. 76, no. 5, pp. 514-518, 2009.

[28] M. B. Kadiiska, B. C. Gladen, D. D. Baird et al., "Biomarkers of oxidative stress study II: are oxidation products of lipids, proteins, and DNA markers of $\mathrm{CCl}_{4}$ poisoning?" Free Radical Biology and Medicine, vol. 38, no. 6, pp. 698-710, 2005.

[29] H. Min, S. Morony, I. Sarosi et al., "Osteoprotegerin reverses osteoporosis by inhibiting endosteal osteoclasts and prevents vascular calcification by blocking a process resembling osteoclastogenesis," Journal of Experimental Medicine, vol. 192, no. 4, pp. 463-474, 2000.
[30] J. M. Liu, H. Y. Zhao, G. Ning et al., "Relationships between the changes of serum levels of OPG and RANKL with age, menopause, bone biochemical markers and bone mineral density in Chinese women aged 20-75," Calcified Tissue International, vol. 76, no. 1, pp. 1-6, 2005.

[31] X.-C. Bai, D. Lu, A.-L. Liu et al., "Reactive oxygen species stimulates receptor activator of NF- $\kappa \mathrm{B}$ ligand expression in osteoblast," Journal of Biological Chemistry, vol. 280, no. 17, pp. 17497-17506, 2005.

[32] C.-L. Shen, V. von Bergen, M.-C. Chyu et al., "Fruits and dietary phytochemicals in bone protection," Nutrition Research, vol. 32, no. 12, pp. 897-910, 2012.

[33] J. C. Tou, "Resveratrol supplementation affects bone acquisition and osteoporosis: pre-clinical evidence toward translational diet therapy," Biochimica et Biophysica Acta-Molecular Basis of Disease, vol. 1852, no. 6, pp. 1186-1194, 2015.

[34] K.-Y. Chin, H. Mo, and I.-N. Soelaiman, "A review of the possible mechanisms of action of tocotrienol-a potential antiosteoporotic agent," Current Drug Targets, vol. 14, no. 13, pp. 1533-1541, 2013.

[35] K.-Y. Chin and S. Ima-Nirwana, "The effects of $\alpha$-tocopherol on bone: a double-edged sword?” Nutrients, vol. 6, no. 4, pp. 14241441, 2014.

[36] R. L. Wolf, J. A. Cauley, M. Pettinger et al., "Lack of a relation between vitamin and mineral antioxidants and bone mineral density: results from the Women's Health Initiative," American Journal of Clinical Nutrition, vol. 82, no. 3, pp. 581-588, 2005.

[37] J. M. Mata-Granados, R. Cuenca-Acebedo, M. D. Luque De Castro, and J. M. Quesada Gómez, "Lower vitamin e serum levels are associated with osteoporosis in early postmenopausal women: a cross-sectional study, Journal of Bone and Mineral Metabolism, vol. 31, no. 4, pp. 455-460, 2013.

[38] H. M. Macdonald, S. A. New, M. H. N. Golden, M. K. Campbell, and D. M. Reid, "Nutritional associations with bone loss during the menopausal transition: evidence of a beneficial effect of calcium, alcohol, and fruit and vegetable nutrients and of a detrimental effect of fatty acids," The American Journal of Clinical Nutrition, vol. 79, no. 1, pp. 155-165, 2004.

[39] K. Michaëlsson, A. Wolk, L. Byberg, J. Ärnlöv, and H. Melhus, "Intake and serum concentrations of $\alpha$-tocopherol in relation to fractures in elderly women and men: 2 cohort studies," The American Journal of Clinical Nutrition, vol. 99, no. 1, pp. 107-114, 2014.

[40] S. Sahni, M. T. Hannan, J. Blumberg, L. A. Cupples, D. P. Kiel, and K. L. Tucker, "Protective effect of total carotenoid and lycopene intake on the risk of hip fracture: a 17-year follow-up from the Framingham Osteoporosis study," Journal of Bone and Mineral Research, vol. 24, no. 6, pp. 1086-1094, 2009.

[41] S. Sahni, M. T. Hannan, D. Gagnon et al., "Protective effect of total and supplemental vitamin $C$ intake on the risk of hip fracture-a 17-year follow-up from the Framingham Osteoporosis Study," Osteoporosis International, vol. 20, no. 11, pp. 18531861, 2009.

[42] A. D. Anastasilakis, D. G. Goulis, S. A. Polyzos et al., "Acute changes in serum osteoprotegerin and receptor activator for nuclear factor- $\kappa \mathrm{B}$ ligand levels in women with established osteoporosis treated with teriparatide," European Journal of Endocrinology, vol. 158, no. 3, pp. 411-415, 2008.

[43] M. D. Evans, R. Singh, V. Mistry, K. Sandhu, P. B. Farmer, and M. S. Cooke, "Analysis of urinary 8-oxo-7,8-dihydro-purine$2^{\prime}$ - deoxyribonucleosides by LC-MS/MS and improved ELISA," Free Radical Research, vol. 42, no. 10, pp. 831-840, 2008. 


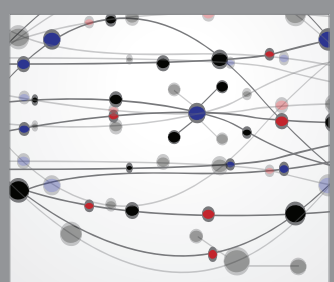

The Scientific World Journal
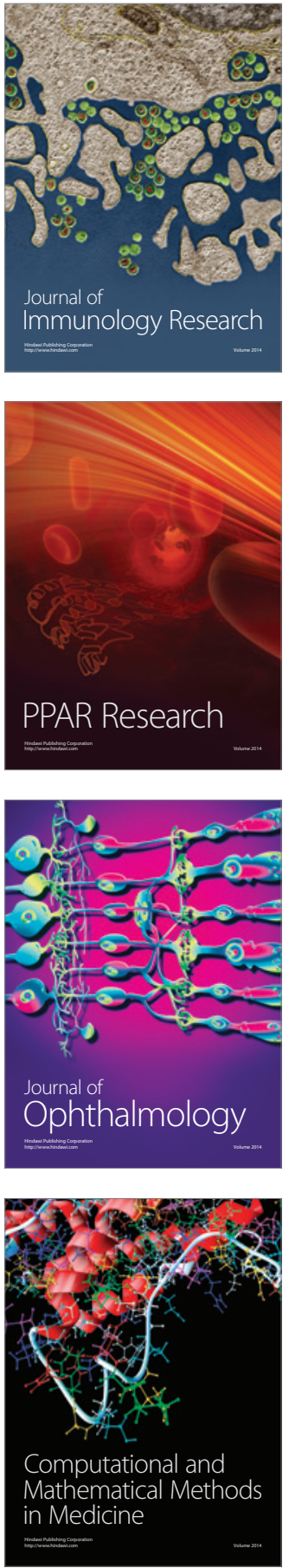

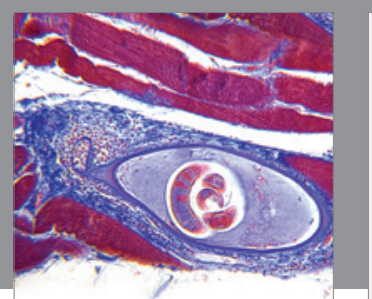

Gastroenterology Research and Practice

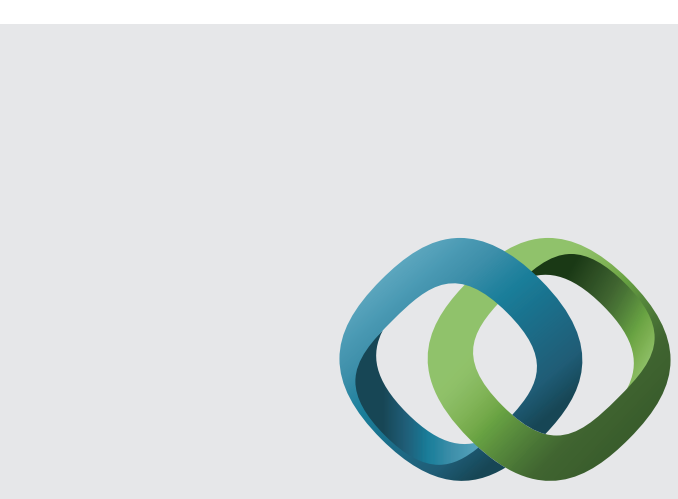

\section{Hindawi}

Submit your manuscripts at

http://www.hindawi.com
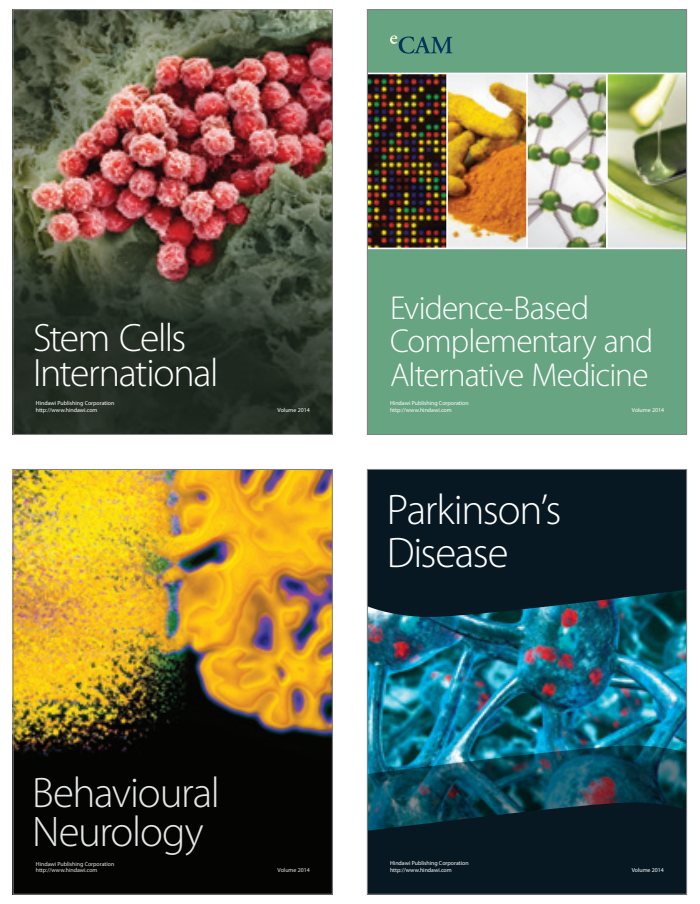
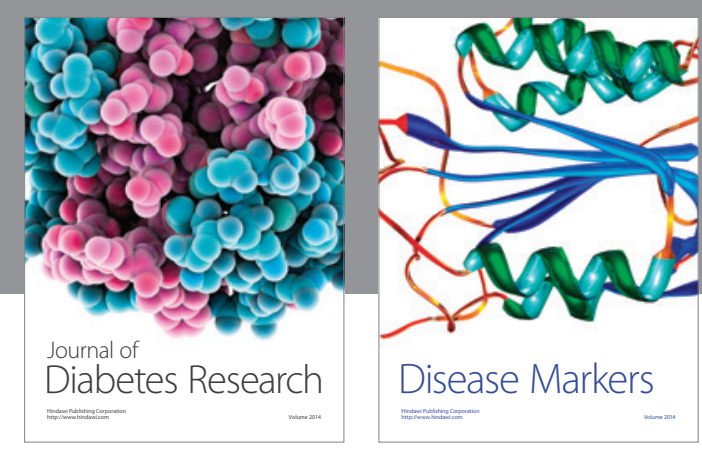

Disease Markers
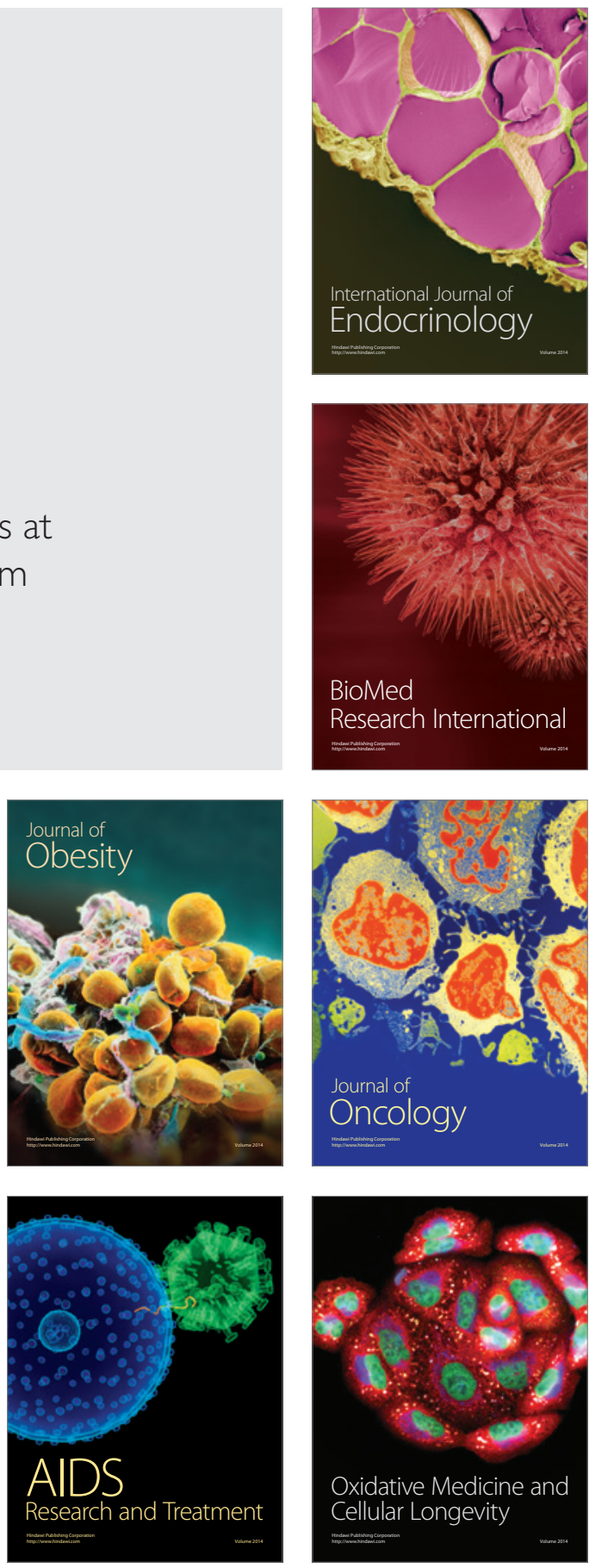